\title{
Comparison of Pharmacist and Physician Managed Annual Medicare Wellness Services
}

\author{
Mary Jean Sewell, PharmD; Daniel M. Riche, PharmD; Joshua W. Fleming, PharmD;
}

Scott S. Malinowski, PharmD; and R. Terry Jackson, MD

\begin{abstract}
BACKGROUND: Medicare Annual Wellness Visits (AWV) are a benefit provided for Medicare beneficiaries to increase focus on wellness and preventive measures. Pharmacists can conduct AWVs, which offers a potential avenue for outpatient revenue generation.
\end{abstract}

PROGRAM DESCRIPTION: To compare a composite of interventions and screenings and revenue generated by a pharmacist with those made by a physician during a subsequent AWV. A report generated through the electronic health record was used to determine AWVs conducted by a pharmacist or 3 participating physicians from December 2013 to March 2016, including revenue generated. Through electronic chart review, documentation was accessed to quantify and categorize the number and types of referrals, health advice, laboratory tests, procedures, vaccinations, and screenings that were recommended during each patient's AWV.

OBSERVATIONS: The pharmacist performed 19 subsequent visits, and the 3 physicians performed 89 subsequent visits. Overall, the composite of interventions and screenings was significantly higher in the pharmacist group than the physician group $(P=0.03)$. More interventions were made in the areas of health advice $(P=0.020)$, vaccine recommendations $(P=0.009)$, and screenings in the pharmacist group $(P<0.001)$. The physicians ordered significantly more laboratory tests per visit $(P<0.001)$. The pharmacist was reimbursed on average $\$ 105$ per visit versus $\$ 99$ per visit for the physicians.

IMPLICATIONS: Pharmacist-provided AWVs are at least comparable to those provided by physicians and offer an additional access point for valuable services for Medicare beneficiaries.

J Manag Care Spec Pharm. 2016;22(12):1412-16

Copyright $\odot 2016$, Academy of Managed Care Pharmacy. All rights reserved.

\section{What is already known about this subject}

Provision of Medicare wellness services is limited at this time. Pharmacists providing Medicare Annual Wellness Visits are able to generate revenue to help support clinical practice.

\section{What this study adds}

Pharmacists provide Medicare wellness services that are at least comparable to those provided by physicians.

Pharmacists receive similar reimbursement for provision of Medicare Annual Wellness Visits as physicians.
I n January 2011, the Affordable Care Act began requiring that Medicare cover Annual Wellness Visits (AWV) in order to increase focus on each patient's wellness and preventive measures. To minimize patient barriers to receiving preventive and screening services, AWVs are provided at no cost to the Medicare beneficiary. An initial AWV can be performed after a patient has been enrolled in Medicare for at least 12 months. Subsequent visits are conducted at least 12 months apart and focus on updating much of the information obtained during the initial AWV

Medicare specifies that AWVs can be provided by other licensed practitioners, including "a physician, qualified nonphysician practitioner, medical professional (including a health educator, registered dietitian, nutrition professional, or other licensed practitioner), or team of such medical professionals who are working under the direct supervision of a physician." Pharmacists are one of the qualified nonphysician practitioners who are authorized to perform AWVs for Medicare patients.

A report by Thomas and Goode (2014) found that 1 pharmacist ( 8 hours per week) performing AWVs in a private family practice office was able to produce significant revenue for the site. ${ }^{2}$ This report focused on financial data rather than data on interventions and screenings. In an academic medical center clinic, 1 pharmacist already working in a clinic added 16 schedule slots per month for AWVs. The average number of interventions was 4.5 per AWV, but revenue data were only estimated based on charges. ${ }^{3}$ Recently, data were reported on interventions, actual revenue, and downstream revenue by a group of pharmacists providing AWVs in an accountable care organization. ${ }^{4}$ The authors reported an average of 5.4 interventions per patient visit. ${ }^{4}$ However, a couple of gaps still exist in the published literature. The only report of interventions and actual revenue by pharmacists providing AWVs has been in an accountable care organization. There are no published data that qualitatively and quantitatively compare AWVs performed by a pharmacist with another health care provider.

AWVs are beneficial for Medicare beneficiaries but are not performed regularly in many provider offices. A poll performed by the John A. Hartford Foundation in 2012, with the goal of determining geriatric patient use of preventative services, surveyed about 1,000 patients aged over 65 years and found that $68 \%$ of patients over age 65 years had never heard of AWVs, and only $17 \%$ had received an AWV. ${ }^{5}$ The time needed to 
complete an AWV is a primary reason that many providers have not regularly incorporated these visits into their practices. The billing code for an initial AWV (G0438) gives the provider an allotted time of 40 minutes, and a subsequent visit (G0439) gives the provider 25 minutes to complete each visit. One study found that AWVs take approximately 73 minutes on average when completed thoroughly. ${ }^{6}$ The use of other qualified nonphysician practitioners, particularly pharmacists, has helped some practices incorporate AWVs without taking physician time from providing other established services.

It is anticipated that by performing AWVs, patients will be more aware of preventive measures that will likely lead to improved health. While the provision of AWVs has demonstrated a viable income source for pharmacist services at multiple sites, ${ }^{7}$ limited data are available about the result of interventions made during AWVs performed by pharmacists, particularly in comparison with other health care professionals.

There is currently no published literature that compares AWVs performed by a pharmacist with any other health care professional. The purpose of this study was to compare the quantity and types of interventions, as well as revenue, from pharmacist-performed and physician-performed AWVs.

\section{Program Description}

This study was a retrospective cohort analysis performed via chart review. The Medicare AWV clinic used in this study is an outpatient service affiliated with the University of Mississippi Medical Center, a large academic medical center. Patients in the Medicare AWV clinic were included if they were seen by the participating pharmacist or 1 of 3 participating physicians under the AWV collaborative protocol agreement from December 2013 to March 2016 for an initial or subsequent Medicare AWV at an outpatient clinic. The pharmacist provided services on 1 half-day per week, as an extension of previously established clinical services. A report generated through the electronic health record (EPIC) was used to determine AWVs conducted by the pharmacist and the 3 participating physicians. This report was used to determine visits conducted and income generated from AWVs by each provider.

The primary objective of this study was to compare a composite of interventions and screenings and revenue generated made by a pharmacist with those made by a physician during a subsequent AWV. Interventions were considered health advice recommendations, referrals, procedures ordered, laboratory tests ordered, and vaccinations recommended. The primary composite was evaluated with and without screenings, since screenings may be considered lower-tier interventions in outcome analysis. Screenings required by Medicare during an AWV include depression, cognition, functional status, and home safety. Revenue is reported as actual reimbursement received, not charges. Other outcomes included number and type of health advice recommendations, referrals made, and procedures ordered during AWVs conducted by each group. The number of referrals and procedures that were attended and individual components of the primary composite were also evaluated. Data parameters collected included the date of service, service provider, code billed, charge, reimbursement, referrals made and number attended, health advice given, laboratory tests ordered, procedures ordered and number completed, vaccinations recommended and number completed, and recommended screenings performed. Data were collected on all visits conducted in the time period by the same investigator, but the outcome analysis only included subsequent visits for the most direct comparison, considering differences in reimbursement and recommendations between initial and subsequent visits.

Through the use of the electronic health record, the investigator accessed notes written by the providers to determine referrals, health advice, laboratory tests, procedures, vaccinations, and screenings that took place or were recommended during each patient's AWV. The electronic health record was searched in order to determine if patients made and attended appointments for the referrals and procedures that were recommended by the providers. This study was approved by the Institutional Review Board.

In Mississippi, pharmacists are able to act under collaborative protocol agreements, allowing them to see patients independently. The pharmacist AWVs that were conducted during this time period were completed in the same office suite as a collaborating physician without the physician being in the patient's room. Most pharmacist patients were referred from a cardiometabolic clinic, while the physician was the primary care provider for the majority of the patients in the physician group.

Number of interventions ( \pm screenings) per visit and financial data were compared using t-tests. Fisher's exact test was used for dichotomous variables. Statistics were reported as means and standard deviations for continuous data and counts, percentages, medians and minimum/maximum for the categorical data. $P$ values less than 0.05 were considered to be statistically significant. All statistical analyses were conducted using Microsoft Excel (Microsoft, Redmond, WA). Other data and results were reported descriptively.

During the time period evaluated, the pharmacist saw 20 patients for AWVs for a total of 37 visits. The trio of physicians saw 75 patients for AWVs, for a combined total of 91 visits. The pharmacist performed 19 subsequent visits, and the 3 physicians performed 89 subsequent visits. Some patients were seen for multiple visits in the time period, accounting for more visits than patients. Since the physicians only had 2 initial AWVs, only subsequent AWVs were reported. The pharmacist performed initial and subsequent visits at similar rates, while the physicians performed primarily subsequent visits. 


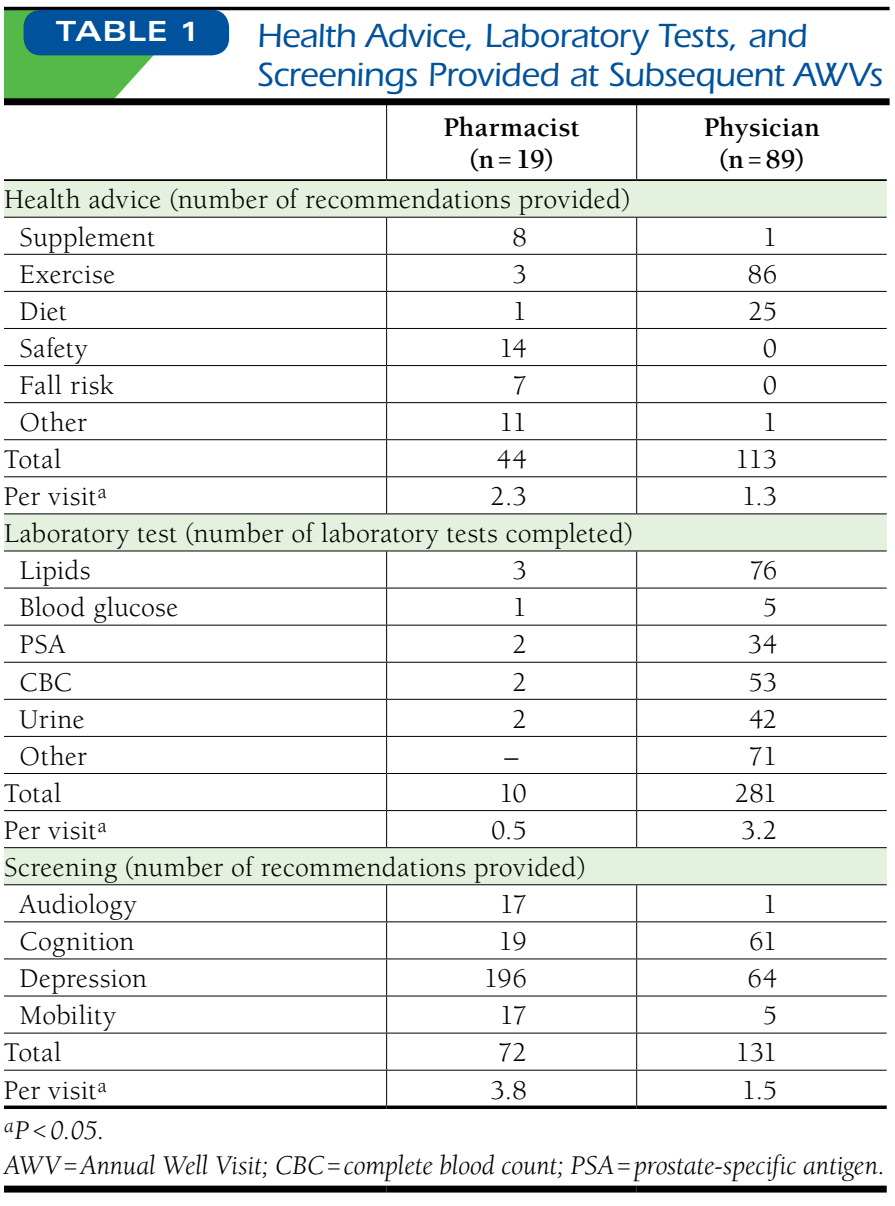

There was not a statistically significant difference in the combined number of interventions made per visit between the pharmacist (4.3 per AWV) and physicians (5.2 per AWV). When the composite of interventions and screenings was analyzed, the pharmacist group had statistically significantly more recommendations per visit compared with the physician group (8.1 vs. 6.6 per AWV; $P=0.03$ ).

Individual components of the composite outcome were also evaluated. In terms of health advice provided, the pharmacist made 2.3 recommendations per visit, compared with 1.3 by physicians $(P=0.020$; Table 1$)$. The pharmacist ordered 1 laboratory test every 2 visits, while the physicians ordered 3.2 per visit $(P<0.001$; Table 1$)$. The pharmacist performed 3.8 screenings per visit, while the physicians performed 1.5 screenings per visit $(P<0.001$; Table 1$)$.

Table 2 shows number of recommended referrals, procedures, and vaccinations. There was 1 referral recommended every 3 visits on average in the pharmacist group, and 1 referral recommended every 10 visits in the physician group. The rate of pharmacist referrals attended was $67 \%$ versus $83 \%$ for physician referrals. Procedures were ordered at the same rate ( 1 in every 3 visits) by both groups, and the vast majority of

\begin{tabular}{|c|c|c|c|c|}
\hline TABLE 2 & \multicolumn{4}{|c|}{$\begin{array}{l}\text { Number of Referrals, Procedures, } \\
\text { and Vaccinations Recommended } \\
\text { and Completed }\end{array}$} \\
\hline & \multicolumn{2}{|c|}{ Pharmacist $(n=19)$} & \multicolumn{2}{|c|}{ Physician $(n=89)$} \\
\hline & 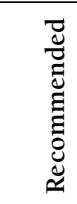 & 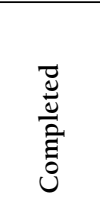 & 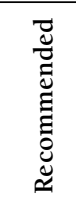 & 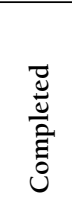 \\
\hline \multicolumn{5}{|c|}{ Referrals (number of referrals provided) } \\
\hline Audiology & 1 & 0 & - & - \\
\hline Specialist & 5 & 4 & 12 & 10 \\
\hline Total/\% complete & 6 & $67 \%$ & 12 & $83 \%$ \\
\hline Per visit & 0.3 & & 0.1 & \\
\hline \multicolumn{5}{|c|}{ Procedures (number of procedures ordered) } \\
\hline DEXA & 2 & 2 & 3 & 3 \\
\hline Mammogram & 2 & 2 & 13 & 8 \\
\hline Colonoscopy & 1 & 1 & 10 & 8 \\
\hline Other & - & - & 8 & 6 \\
\hline Total/\% complete & 5 & $100 \%$ & 34 & $74 \%$ \\
\hline Per visit & 0.3 & & 0.4 & \\
\hline \multicolumn{5}{|c|}{ Vaccines (number of procedures recommended) } \\
\hline PPSV23 & 3 & 3 & 3 & 2 \\
\hline PCV13 & 1 & 1 & 2 & 2 \\
\hline Zoster & 7 & 1 & 4 & 2 \\
\hline Influenza & - & - & 7 & 5 \\
\hline Tdap & 4 & 1 & 2 & 2 \\
\hline Hep B & 1 & 0 & - & - \\
\hline Total/\% complete & 16 & $37 \%$ & 18 & $72 \%$ \\
\hline Per visit ${ }^{\mathrm{a}}$ & 0.8 & & 0.2 & \\
\hline
\end{tabular}

${ }^{a} P<0.01$.

DEXA = Dual-energy X-ray absorptiometry; Hep B=hepatitis $B ; P C V 13=$ pneumococcal conjugate vaccine 13; PPSV23= pneumococcal polysaccharide vaccine 23;

Tdap= tetanus-diphtheria-pertussis

procedures ordered by either group were attended by patients. The pharmacist was 4 times more likely to recommend a vaccination per visit ( 0.8 vs. 0.2 per visit; $P=0.009)$; however, there was a low completion rate in the pharmacist group of $37 \%$ compared with that of the physicians at $72 \%$.

Financial impact was evaluated based on subsequent visits only, which were billed at approximately $\$ 153$ per visit. The pharmacist billed $\$ 2,601$ and was reimbursed $\$ 1,785$ ( $\$ 105$ per visit). The physicians billed $\$ 13,005$ and were reimbursed $\$ 8,866$ ( $\$ 99$ per visit). There was not a statistically significant difference in the reimbursement rate per visit between the pharmacist and physician groups.

\section{Observations}

The primary objective (a composite of interventions and screenings) differed between groups. Many of the provided screenings led to interventions (e.g., referral for hearing aids); therefore, screenings were considered applicable to the primary objective of this study. The pharmacist provided recommended 
screenings for all but 2 patients (neither were able to physically complete the screenings because of other conditions). There was less consistency in number of physician-performed screenings per visit, which typically occurred as a component of a standard review of systems or physical exam.

Health advice recommendations are encouraged as part of AWVs. ${ }^{1}$ The pharmacist made a variety of health advice recommendations based on need identified during AWVs that are listed in Table 1. Physicians consistently made health advice recommendations, but most appeared to be part of their note template (specifically diet and exercise). A limitation to the nature of retrospective data collection is that other recommendations could have been made by a provider but were not necessarily documented.

Referrals made as part of AWVs are based on needs that arise during patient interactions. Most of the referrals were made to specialists (e.g., cardiology and dermatology). Health maintenance procedures were appropriately ordered during AWVs regardless of service provider.

Medicare neither requires nor encourages laboratory tests to be performed as part of an AWV. ${ }^{1}$ Most laboratory tests are not covered as a component of AWVs. The physicians ordered significantly more laboratory tests on a per visit basis. During the course of an AWV, it is common for patients to introduce a nonwellness compliant or chronic disease condition to their physicians. Therefore, it would be expected that physicians will order more laboratory tests over the course of any patient care visit, regardless of the type of visit charged. Physicians are able to recuperate costs for effort spent during evaluation $\&$ management (E/M) service beyond AWV (known as a modifier 25), while pharmacists are not able to independently submit an additional E/M service claim. In this dataset, modifier 25 codes were very infrequent ( $<5 \%$ of submitted charges). ${ }^{8}$ Supplemental (non-AWV specific) laboratory testing was not supported generally by the physician/pharmacist collaborative protocol agreement in place. When applicable, the pharmacist recommended that patients follow up with their primary care physicians for specific laboratory monitoring. Additionally, the pharmacist AWVs were based on referrals from a primary care physician. Therefore, patients were likely to have had laboratory tests completed by their primary care physicians before the AWV. These reasons may explain the discrepancy in laboratory monitoring between the groups.

Vaccines are also recommended as part of the health maintenance included in AWVs. There was a low completion rate of vaccines in both groups, particularly in the pharmacist group. During the time period of this study, there were a couple of barriers to patients receiving vaccines, specifically lack of vaccine coverage by the patient's insurance and lack of access to vaccines at the clinic site. As a limitation of the retrospective nature of this study, patients could have received immunizations that were recommended by a provider in either group outside of our health system; therefore, vaccine completion rates should be interpreted as a conservative estimate.
In comparison with previously published data on pharmacist-delivered Medicare AWV, this study appears to be similar. In a private family practice office, reimbursement for initial AWVs were $\$ 163.51$ per patient. ${ }^{2}$ Our reported reimbursement was lower because of the exclusion of initial visits for comparative analysis. Our initial visit reimbursement was similar to that reported previously. Based on intervention reports in a large, academic medical center (4.5 per AWV) and in an accountable care organization (5.4 per AWV), our results are similar in average number of interventions plus or minus screenings (4.3 and 8.1 per AWV, respectively). ${ }^{3,4}$ Our data are the only report of interventions and actual revenue by pharmacists providing AWVs outside of an accountable care organization.

\section{Limitations}

In addition to the retrospective design, there are several limitations to this study. The overall sample size was small, although AWVs are infrequently performed in general. During data collection, inconsistencies were noted in the pharmacist and physician documentation. The physicians appeared to have used a templated note suited for many types of patient visits, while the pharmacist used a specific note template for AWVs. While the note template may have led to increased interventions and screenings, the AWV template was significantly more time intensive. Since physician time is at a premium in the ambulatory setting, a nonphysician practitioner using an extensive AWV template may be more cost-effective from an institutional perspective. Finally, data were collected for all visits conducted during the study period, but because of the small number of initial visits conducted in the physician group $(n=2)$, initial visits were not analyzed in this comparison.

\section{Implications}

A pharmacist provides quantifiably similar AWVs to that of a physician in terms of interventions. Higher rates of health advice recommendations, vaccination recommendations, and screenings were observed in AWVs conducted by a pharmacist, while physicians had a higher rate of ordering laboratory tests. By implementing pharmacist provision of AWVs, an additional access point for valuable services that are currently underused is made available for Medicare beneficiaries.

This study was not intended to be a qualitative comparison, rather, it was intended as a first step towards demonstrating that, in terms of quantifiable outcomes, pharmacists are not inferior to physicians in conducting AWVs. Potential implications of this data show that physicians can incorporate pharmacists into their practices to off-load some of the AWV time burden with the comfort of knowing that a pharmacist can provide AWVs similarly with identical reimbursement. 


\section{Authors}

MARY JEAN SEWELL, PharmD; JOSHUA W. FLEMING, PharmD; and SCOTT S. MALINOWSKI, PharmD, The University of Mississippi School of Pharmacy, Jackson. DANIEL M. RICHE, PharmD, The University of Mississippi School of Pharmacy, and The University of Mississippi School of Medicine, Jackson. R. TERRY JACKSON, MD, The University of Mississippi School of Medicine, Jackson.

AUTHOR CORRESPONDENCE: Daniel M. Riche, PharmD, Associate Professor of Medicine, University of Mississippi Medical Center, 2500 N. State St., Jackson, MS 39216. Tel.: 601.984.2640; Fax:601.984.2751; E-mail: driche@umc.edu.

\section{DISCLOSURES}

There was no financial contribution to this study. Riche reports participation in the Speaker's Bureau for Merck and the Speaker's Bureau and Advisory Board for Novo Nordisk. The authors have no other conflicts of interest to report pertinent to this research. This data has not been previously published in any other location.

Richie, Sewell, Malinowski, Jackson, and Fleming were involved in study design and manuscript preparation/approval. Jackson was involved in data collection, and Richie and Sewell were involved in data collection and data analysis. Sewell and Richie had full access to all the data in the study and take responsibility for the integrity of the data and the accuracy of the data analysis.

\section{ACKNOWLEDGMENTS}

The authors acknowledge Michelle Horn, MD, and Muhammah Ullah, MD, for assisting with patient enrollment.

\section{REFERENCES}

1. Centers for Medicare $\&$ Medicaid Services. The ABCs of the Annual Wellness Visit (AWV). Medicare Learning Network. January 2015. Available at: https://www.cms.gov/Outreach-and-Education/Medicare-LearningNetwork-MLN/MLNProducts/downloads/AWV_chart_ICN905706.pdf. Accessed October 23, 2016.

2. Thomas MH, Goode JV. Development and implementation of a pharmacist-delivered Medicare annual wellness visit at a family practice office. J Am Pharm Assoc. 2014;54(4):427-34.

3. Warshany K, Sherrill $\mathrm{CH}$, Cavanaugh J, et al. Medicare annual wellness visits conducted by a pharmacist in an internal medicine clinic. Am J Health Syst Pharm. 2014;71(1):44-49.

4. Alhossan A, Kennedy A, Leal S. Outcomes of annual wellness visits provided by pharmacists in an accountable care organization associated with a federally qualified health center. Am J Health Syst Pharm. 2016;73(4):225-28.

5. John A. Hartford Foundation. National poll: low cost, lifesaving services missing from most older patients' health care. April 24. 2012. Available at: http://www.jhartfound.org/images/uploads/resources/120420_JAHF_poll_ release_FINAL.pdf. Accessed October 23, 2016.

6. Shilliday BB. Medicare wellness visits - a new reimbursable service model for pharmacists. ASHP: Section of Ambulatory Care Practitioners. In the Spotlight. June 2012. Available at: https://www.ashp.org/DocLibrary/MemberCenter/ SACP/Spotlight/Spotlight-June-2012.aspx. Accessed October 23, 2016.

7. Park I, Sutherland SE, Ray L, Wilson CG. Financial implications of pharmacist-led Medicare annual wellness visits. J Am Pharm Assoc. 2014;54(4):435-40.

8. American College of Physicians. How to bill Medicare's Annual Wellness Visit. Available at: https://www.acponline.org/practice-resources/businessresources/payment/medicare/how-to-bill-medicares-annual-wellness-visitawv. Accessed October 23, 2016. 